\title{
Transmitting Performance Evaluation of ASICs for CMUT-Based Portable Ultrasound
} Scanners

Llimos Muntal, Pere; Diederichsen, Søren Elmin; Jørgensen, Ivan Harald Holger; Jensen, Jørgen Arendt; Thomsen, Erik Vilain

\section{Published in:}

Proceedings of 2017 IEEE International Ultrasonics Symposium (IUS)

Link to article, DOI:

10.1109/ULTSYM.2017.8092913

Publication date:

2017

Document Version

Peer reviewed version

Link back to DTU Orbit

Citation (APA):

Llimos Muntal, P., Diederichsen, S. E., Jørgensen, I. H. H., Jensen, J. A., \& Thomsen, E. V. (2017). Transmitting Performance Evaluation of ASICs for CMUT-Based Portable Ultrasound Scanners. In Proceedings of 2017 IEEE International Ultrasonics Symposium (IUS) IEEE. https://doi.org/10.1109/ULTSYM.2017.8092913

\section{General rights}

Copyright and moral rights for the publications made accessible in the public portal are retained by the authors and/or other copyright owners and it is a condition of accessing publications that users recognise and abide by the legal requirements associated with these rights.

- Users may download and print one copy of any publication from the public portal for the purpose of private study or research.

- You may not further distribute the material or use it for any profit-making activity or commercial gain

- You may freely distribute the URL identifying the publication in the public portal 


\title{
Transmitting Performance Evaluation of ASICs for CMUT-Based Portable Ultrasound Scanners
}

\author{
Pere Llimós Muntal*, Søren Elmin Diederichsen ${ }^{\dagger}$, Ivan H. H. Jørgensen*, Jørgen A. Jensen ${ }^{\ddagger}$, Erik V. Thomsen ${ }^{\dagger}$, \\ ${ }^{*}$ Department of Electrical Engineering, Electronics Group \\ ${ }^{\dagger}$ Department of Micro- and Nanotechnology \\ $\ddagger$ Department of Electrical Engineering, Center for Fast Ultrasound Imaging \\ Technical University of Denmark, Kgs. Lyngby 2800, Denmark
}

\begin{abstract}
Portable ultrasound scanners (PUS) have, in recent years, raised a lot of attention, as they can potentially overcome some of the limitations of static scanners. However, PUS have a lot of design limitations including size and power consumption. These restrictions can compromise the image quality of the scanner. In order to overcome these restrictions, application specific integrated circuits (ASICs) are needed to implement the electronics. In this work, a comparative study of the transmitting performance of a capacitive micromachined ultrasonic transducer (CMUT) driven by a commercial generic ultrasound transmitter and an ASIC optimized for CMUT-based PUS is presented. A single CMUT element is pulsed with a $1 \%$ duty cycle at a frequency of $5 \mathrm{MHz}$. The DC bias voltage is $80 \mathrm{~V}$ and the pulsing voltage is $20 \mathrm{~V}$. The acoustic performance is assessed by comparing the ultrasonic signals measured with a hydrophone both in the time and frequency domains. The difference in normalized signal amplitude evaluated at the center frequency of the CMUT is $-1.9 \mathrm{~dB}$ and the measured bandwidth is equivalent. The ASIC consumes only $1.3 \%$ of the total power consumption used by the commercial transmitter.
\end{abstract}

\section{INTRODUCTION}

Nowadays, traditional static ultrasound scanners are large devices that provide high quality imaging and a wide range of scanning possibilities. In order to achieve that, these scanners require a large amount of complex electronic circuits to implement the scanner functionality. Due to the high complexity, these circuits are very power consuming, which forces the scanner to be connected to the AC mains. As a result of these characteristics, static ultrasound scanners are large devices with limited transportability. Furthermore, their high cost limits the amount of scanners available per hospital, and therefore, reduces their accessibility.

In recent years, portable ultrasound scanners (PUS) have emerged due to their potential of solving some of the limitations of static scanners. These devices are handheld sized, and they have a much lower cost per unit, which enhances both the transportability and accessibility of ultrasound scanning.

However, these advantages also impose very severe restrictions on the scanner design. Due to the small size of PUS, the amount of electronic circuitry that can be contained in the scanner is constrained. Furthermore, the maximum heat that can be dissipated within a handheld device sets another limitation to the maximum power consumption of the system. Moreover, since PUS are not connected to the AC mains, their power consumption is also restricted by the power source (i.e. battery, USB, etc.).

These restrictions have an impact on the image quality of the scanner $\left(Q_{i m}\right)$, which makes the design of PUS very challenging. The design goal is to implement a scanner with a usable $Q_{i m}$ within very strict area and power budgets.

In order to fully utilize the available area and power budgets to achieve the best $\mathrm{Q}_{\mathrm{im}}$, application specific integrated circuits (ASICs) are required for the electronics. The ASICs are optimized to drive a specific transducer while minimizing the area and power consumption, which is a key target for PUS.

In this work, and for the purpose of implementing compact probes, capacitive micromachined ultrasonic transducers (CMUTs) are used due to high compatibility and integration with ASIC complementary metal oxide semiconductor (CMOS) fabrication processes [1]-[4].

The goal of this work is to assess the impact of the area and power consumption limitations of PUS on the transmitting acoustic performance of the scanner. For this purpose, a comparison between a commercial generic ultrasound transmitter and an ASIC transmitter optimized for CMUT-based PUS is presented.

\section{Portable Ultrasound Scanner System}

\section{A. Image Quality Tradeoffs of PUS}

Ultrasound scanners are composed of several transducer elements, a transmitting circuit (Tx) and a receiving circuit (Rx), Fig. 1. In transmitting mode, the Tx drives the transducer elements with high-voltage signals to generate the ultrasonic waves. In receiving mode, the transducers capture the reflected ultrasonic waves inducing electrical signals that are conditioned by the $\mathrm{Rx}$ circuit. The $\mathrm{Q}_{\mathrm{im}}$ obtained by the scanner depends on the characteristics of the transducers, the driving capabilities of the Tx, the signal-to-noise ratio (SNR) of the $\mathrm{Rx}$ and the algorithms used to generate the image. Each of these factors can limit the maximum image quality that the system can achieve ( $\left.\mathrm{Q}_{\mathrm{im}, \mathrm{max}}\right)$.

Typically, in static scanners, the Tx and Rx electronics are over-designed to assure that they do not limit $\mathrm{Q}_{\text {im,max }}$. This approach costs area and power, which are not restrictive factors in static scanners. Nonetheless, PUS have very limited budgets both in terms of area and power consumption. As a result, 


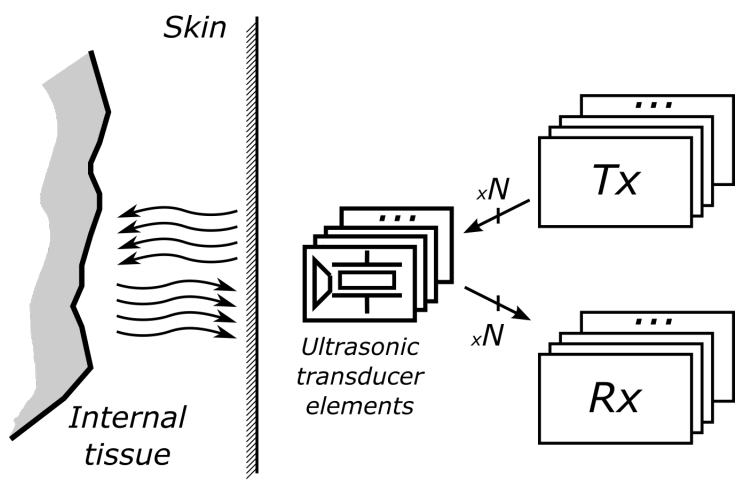

Fig. 1. Structure of an N-channel ultrasound scanner, containing the ultrasonic transducers, the transmitting circuits (Tx) and the receiving circuits (Rx).

the Tx and Rx performance needs to be compromised to fit those budgets, which can have an impact on $\mathrm{Q}_{\text {im,max. }}$. This paper focuses on the impact of the Tx performance on the transmitting acoustic performance of the CMUT, which has an impact on the overall $\mathrm{Q}_{\mathrm{im}, \max }$ of the scanner.

\section{B. Capacitive Micromachined Ultrasonic Transducers (CMUTs)}

Conventionally, ultrasonic transducers for medical imaging have been based on a piezoelectric principle of operation, i.e. a piezoelectric material deforms when an AC signal is applied and thereby creating pressure waves, and vice versa. The emerging CMUT technology, however, relies on an electrostatic principle of operation, as the energy transduction is based on the vibration of a movable top electrode relative to a fixed bottom electrode [5]. A major difference is, therefore, the fact that the CMUT element is less bulky than its piezoelectric counterpart. As a result, the CMUT generally has a higher bandwidth due to its relatively smaller inertia [6]. Other advantages of the CMUT include higher axial resolution, excellent thermal properties and the potential ease of compatibility with ASICs.

A 192-element, $4.7 \mathrm{MHz}$ linear array has been fabricated using a local oxidation of silicon process combined with a direct wafer fusion bonding [7]. Each element measures $6 \mathrm{~mm}$ by $196 \mu \mathrm{m}$ and consists of 450 circular cells with a radius of $23.54 \mu \mathrm{m}$. The cells are hexagonally closed-packed, resulting in a fill factor of $67 \%$ and a total element capacitance of approximately $18 \mathrm{pF}$. A principle sketch of the CMUT is seen in Fig. 2.

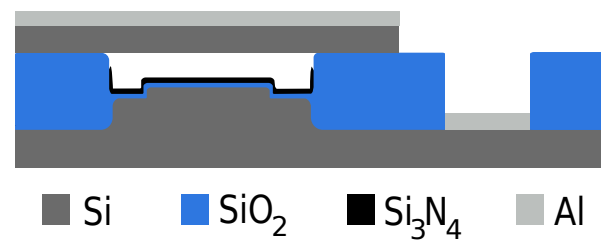

Fig. 2. Illustration showing a cross-sectional view of one CMUT cell (not to scale). The CMUT chip used in this work has 460 cells in each element.

\section{Transmitting circuitry (Tx)}

Apart from the characteristics described in the previous subsection, every CMUT requires a specific driving strength to operate. This driving strength is the slew rate (SR) of the high-voltage driving signals. The area and power consumption of the Tx will increase with the SR required, therefore, an excessive driving strength translates into excessive energy consumed. As a result, the Tx has to be designed for a specific CMUT with an optimal SR. For the CMUTs used in this work, this $\mathrm{SR}$ is $2 \mathrm{~V} / \mathrm{ns}$.

Once all the driving characteristics of the CMUT have been defined an application specific integrated circuit (ASIC) containing a custom designed Tx was designed. A micrograph of the silicon die fabricated is shown in Fig. 3. The ASIC was fabricated in a $0.35 \mu \mathrm{m}$ high-voltage process and occupies a die area of $0.9 \mathrm{~mm}^{2}$. The Tx can be implemented with different structures and topologies such as the ones found in [8]-[10]. For this work, the structures and topologies in [9] were used.

\section{Acoustic Performance Measurements}

\section{A. Comparison Approach}

The aim of this paper is to assess the effect of the area and power limitations of PUS in the transmitting acoustic performance. Therefore, the custom designed ASIC is compared with a commercially available transmitting circuit for ultrasound applications. Commercially available transmitters have a very large SR and a lot of flexibility (frequencies, voltages, etc.) in order to be able to drive any possible transducer. As it was stated before, that comes with an area and power consumption cost. Contrary, the ASIC has been custom designed for a specific transducer and it has an optimally designed performance, which minimizes the die area and power consumption.

In this work, the ASIC is compared with the commercial ultrasound transmitter HDL6V5582. This transmitter has the option of using eight channels with a three-level voltage output and is capable of providing a specified pulse of high symmetry.

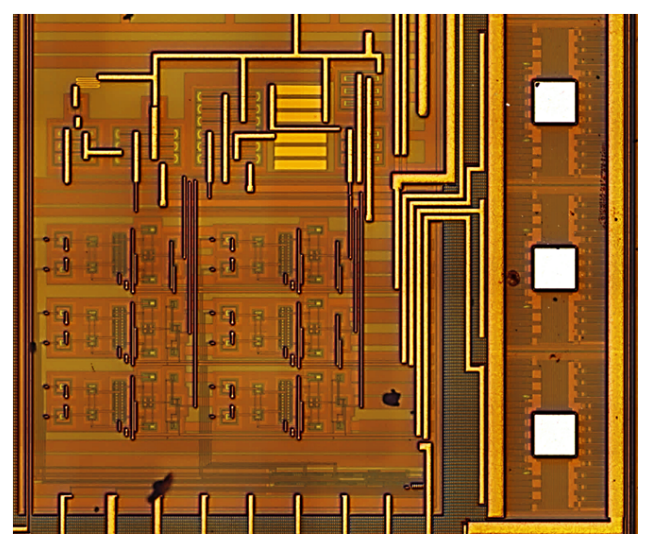

Fig. 3. Micrograph of the fabricated ASIC silicon die. 


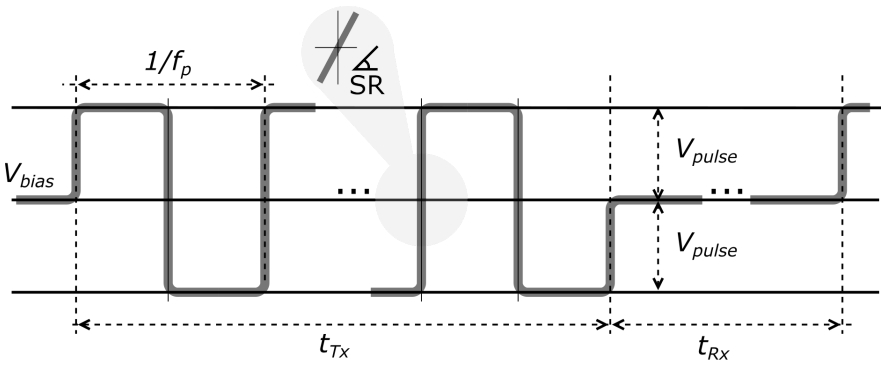

Fig. 4. Pulsing characteristics to drive the CMUT used for both the ASIC and the HDL6V5582.

The HDL6V5582 provides an output voltage of up to $\pm 100 \mathrm{~V}$, an output current of $1.8 \mathrm{~A}$, and operates at frequencies up to $20 \mathrm{MHz}$.

For the purpose of achieving a fair comparison, the same CMUT described in the previous section was used. Furthermore, the pulsing characteristics of the HDL6V5582 were set to the same as the ASIC, which are shown in Fig. 4. The pulsing frequency, $f_{c}$, is set to $5 \mathrm{MHz}$, the DC bias voltage, $V_{\text {bias }}$, to $80 \mathrm{~V}$ and the pulsing voltage amplitude, $V_{\text {pulse }}$, to $20 \mathrm{~V}$. The transmitting time, $t_{T x}$, and receiving time, $t_{R x}$, are set to achieve a $1 \%$ transmitting duty cycle. The CMUT will be driven with the ASIC and the HDL6V5582, and the acoustic performance of the transducer will be compared.

\section{B. Measurement Setup}

A picture of the measurement setup is shown in Fig. 5. A CMUT array is mounted on a printed circuit board with aluminum wires ensuring electrical connection to the CMUT. A polydimethylsiloxane polymer is encapsulating the CMUT and wires to enable measurements in immersion. An Optel hydrophone (Optel Ultrasonic Technology, Poland) is placed at a distance of $1 \mathrm{~cm}$ from the CMUT surface and aligned with a XY stage to obtain the highest signal possible. The setup includes power supplies for biasing the CMUT as well as driving electronics.

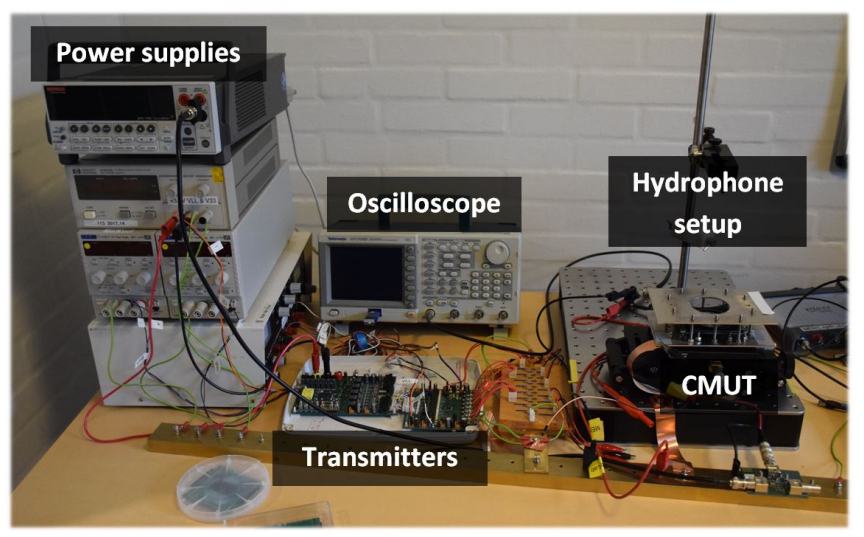

Fig. 5. Picture of the measurement setup used for the comparison.

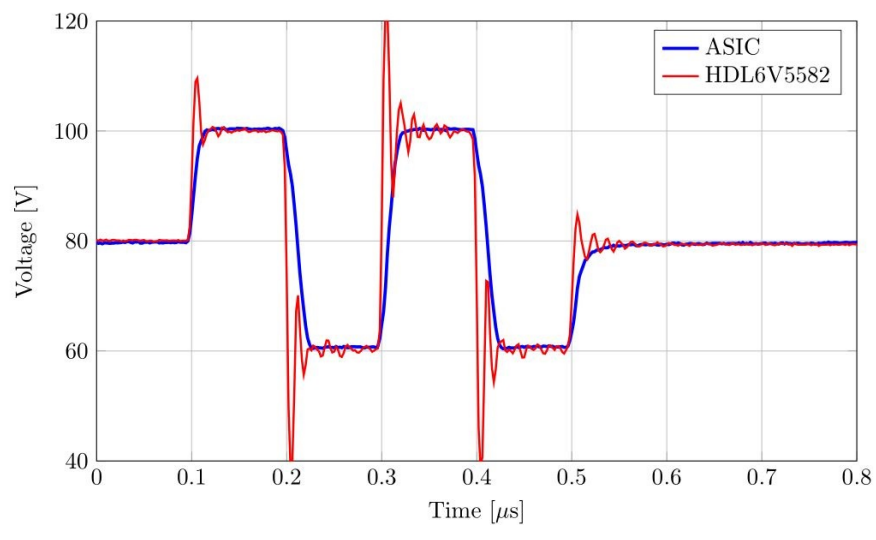

Fig. 6. Transmitting voltage pulses measured at the output terminal of the ASIC and HDL6V5582.

\section{Measurements and Results}

One CMUT element is driven with a square pulse using the ASIC and the HDL6V5582. The square pulse measured at the output terminal of each device is show in Fig. 6. As it can be seen the driving strength and SR of the HDL6V5582 is higher than the ASIC, which has been custom designed to $2 \mathrm{~V} / \mathrm{ns}$. Due to this high SR, the voltage shapes generated with the HDL6V5582 produce some oscillations. The ultrasonic signals received with the hydrophone using the excitation pulses in Fig. 6 are shown in Fig. 7. The signals received for both transmitters are similar, however, it can be observed that the amplitude of the first three oscillations is higher for the HDL6V5582. This is due to its higher SR that drives the CMUT with higher energy. However, the impact of these oscillations on the signal quality can be assessed more accurately through the signal frequency spectrum.

The frequency spectrum of the received signal normalized to the maximum hydrophone voltage is shown in Fig. 8. As it can be seen, the acoustic performance achieved with the ASIC is comparable to the one achieved with the HDL6V5582, with a minor amplitude difference of $-1.9 \mathrm{~dB}$ at the center frequency of the CMUT. Furthermore, the bandwidth measured is not limited by the ASIC.

The power consumption of the ASIC is measured to be $5.8 \mathrm{~mW}$ and the measured power used by the HDL6V5582 is $447.6 \mathrm{~mW}$, i.e. the ASIC consumes $1.3 \%$ of the power used by the HDL6V5582. The ASIC achieves the goal of significantly reducing the power consumption without compromising the transmitting acoustic performance of the scanner.

Furthermore, there is a lot of area reduction potential using ASICS, since a commercial component such as HDL6V5582 has 8 channels, and with the area results achieved with the ASIC, $0.9 \mathrm{~mm}^{2}$, more channels could be included per integrated circuit package. The results presented in this work show a high potential for the future of PUS, since reducing power and area is needed to make them viable to implement.

The next step on the assessment of the impact of the area and power consumption limitations of PUS is to perform a similar comparison for the Rx circuitry. 


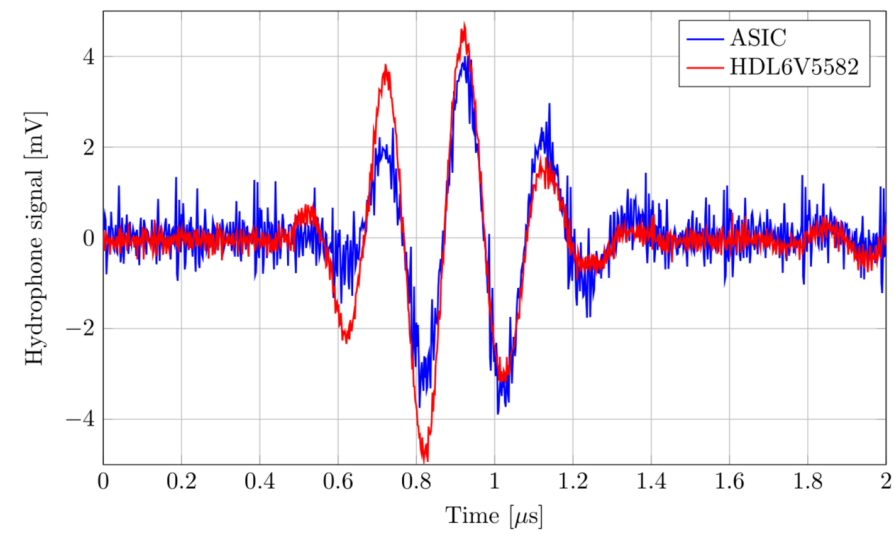

Fig. 7. Signal received with the hydrophone using: a) ASIC and b) HDL6V5582.

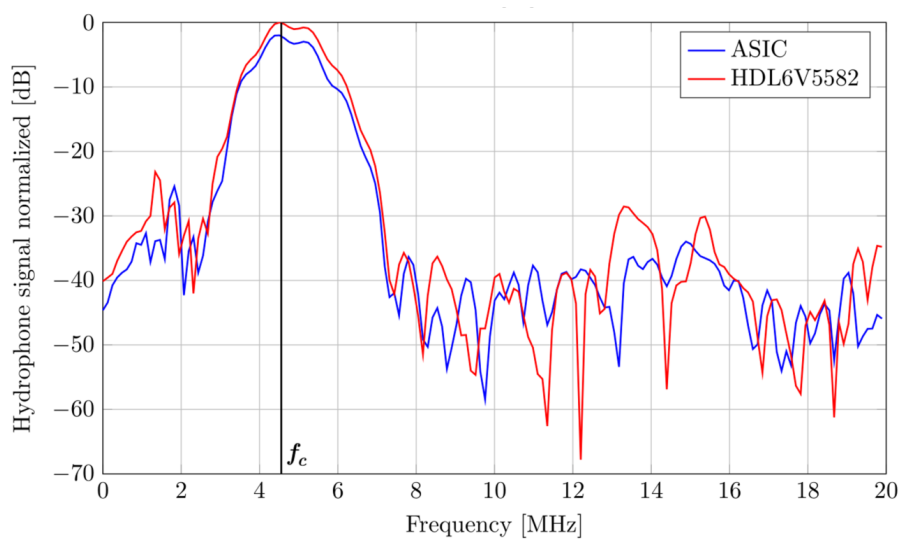

Fig. 8. Frequency spectrum of the signal received with the hydrophone using: a) ASIC and b) HDL6V5582.

\section{CONCLUSION}

In this work, the impact of the area and power consumption limitations of portable ultrasound scanners on the transmitting acoustic performance is assessed. In order to do that, an ASIC, containing a custom designed transmitter, is fabricated in a $0.35 \mu \mathrm{m}$ high-voltage process. The circuit occupies a die area of $0.9 \mathrm{~mm}^{2}$ for one channel and transmits with a slew rate optimized for a specific CMUT of $2 \mathrm{~V} / \mathrm{ns}$. The ASIC is compared to a commercially available ultrasound transmitter, the HDL6V5582. Both transmitters are used to pulse the same CMUT with the same pulsing frequency of $5 \mathrm{MHz}$, duty cycle of $1 \%$, DC bias voltage of $80 \mathrm{~V}$ and pulsing voltage amplitude of $20 \mathrm{~V}$. The transmitting acoustic performance of the CMUT pulsed with the two transmitters is measured using a hydrophone. The ASIC achieves a comparable performance with a minor amplitude difference of $-1.9 \mathrm{~dB}$ at the center frequency of the CMUT, while not limiting the bandwidth of the measurements. The total power consumption of the ASIC is $1.3 \%$ of the total power consumption used by the HDL6V5582.

\section{REFERENCES}

[1] P.-C. Eccardt, K. Niederer, T. Scheiter, and C. Hierold, "Surface micromachined ultrasound transducers in CMOS technology," in Ultrasonics Symposium, 1996. Proceedings., 1996 IEEE, vol. 2. IEEE, 1996, pp. 959-962.

[2] M. Hochman, J. Zahorian, S. Satir, G. Gurun, T. Xu, M. Karaman, P. Hasler, and F. L. Degertekin, "CMUT-on-CMOS for forward-looking IVUS: Improved fabrication and real-time imaging," in 2010 IEEE International Ultrasonics Symposium. IEEE, 2010, pp. 555-558.

[3] G. Gurun, P. Hasler, and F. L. Degertekin, "A 1.5-mm diameter single-chip CMOS front-end system with transmit-receive capability for CMUT-on-CMOS forward-looking IVUS," IEEE International Ultrasonics Symposium, IUS, pp. 478-481, 2011.

[4] — " "Front-end receiver electronics for high-frequency monolithic CMUT-on-CMOS imaging arrays," IEEE Transactions on Ultrasonics, Ferroelectrics, and Frequency Control, vol. 58, no. 8, pp. 1658-1668, 2011.

[5] M. Haller and B. Khuri-Yakub, "A surface micromachined electrostatic ultrasonic air transducer," in Ultrasonics Symposium, 1994. Proceedings., 1994 IEEE, vol. 2. IEEE, 1994, pp. 1241-1244.

[6] I. Ladabaum, B. Khuri-Yakub, D. Spoliansky, and M. Haller, "Micromachined ultrasonic transducers (MUTs)," in Ultrasonics Symposium, 1995. Proceedings., 1995 IEEE, vol. 1. IEEE, 1995, pp. 501-504.

[7] K. Park, H. Lee, M. Kupnik, O. Oralkan, and B. Khuri-Yakub, "Fabricating capacitive micromachined ultrasonic transducers with direct waferbonding and LOCOS technology," in Micro Electro Mechanical Systems. IEEE 21st International Conference on. IEEE, 2008, pp. 339-342.

[8] K. Chen, H. S. Lee, A. P. Chandrakasan, and C. G. Sodini, "Ultrasonic imaging transceiver design for cmut: A three-level 30-vpp pulse-shaping pulser with improved efficiency and a noise-optimized receiver," IEEE Journal of Solid-State Circuits, vol. 48, no. 11, pp. 2734-2745, 2013.

[9] P. Llimós Muntal, D. Ø. Larsen, I. H. H. Jørgensen, and E. Bruun, "Integrated reconfigurable high-voltage transmitting circuit for CMUTs," Analog Integrated Circuits and Signal Processing, vol. 84, no. 3, pp. 343-352, sep 2015.

[10] P. Llimós Muntal, D. Ø. Larsen, K. U. Færch, I. H. H. Jørgensen, and E. Bruun, "High-voltage integrated transmitting circuit with differential driving for CMUTs," Analog Integrated Circuits and Signal Processing, vol. 89, no. 1, pp. 25-34, 2016. 